\title{
Discrete breathers of different symmetry in monoatomic 2D Morse crystal
}

\author{
S.Yu. Fomin ${ }^{1, \dagger}$, E.A. Korznikova ${ }^{2}$ \\ †seryogafom@hotmail.com \\ ${ }^{1}$ Ufa State Aviation Technical University, Ufa \\ ${ }^{2}$ Institute of metals superplasticity problems
}

\begin{abstract}
A considerable growth of attention to the investigation of nonlinear, spatially localized vibrational modes called discrete breathers (DB) in defect free lattices is observed in many areas of modern physics. The existence of DB in crystal lattices is provided by the anharmonicity of the interatomic forces, leading to a dependence of vibration frequencies of atoms on their amplitudes. It has been shown that various crystals can support DB having different properties. The study of DB and their effect on crystal properties is a subject for numerous investigations. An important problem is to find the initial conditions to excite a DB. Crystals with Morse interaction potential are convenient objects for investigation of different DB types due to their simplicity. In the present study we focus on the analysis of the two-dimensional monoatomic crystal with Morse interaction potential and reveal the possibility of existence of immobile high symmetry DB in addition to the described earlier moving DB localized in a close-packed atomic row. For excitation of both types of DB similar approach is used, which is the application of a bell-shaped functions on a short-wavelength phonon mode in the nonlinear regime. Amplitude - frequency dependencies of $\mathrm{DB}$ central atoms is calculated for both types of DB. In both cases DB frequency lies above the phonon spectrum and increases with the amplitude.
\end{abstract}

Keywords: two-molecular dynamics, Morse interaction potential, discrete breather

\section{Introduction}

Discrete breathers (DB) - is spatially localized largeamplitude vibrational mode in a defect-free nonlinear lattice $[1-3]$. Over the past ten years investigation of DB in different materials has been the subject of numerous works, including theoretical and experimental ones [4-11], performed by means of molecular dynamics [15-45] an ab initio approaches [12-14].

The possibility of DB existence is provided by anharmonicity of the interatomic forces, leading to a variation of atoms oscillation frequency upon amplitude. DB has the oscillation frequency out of the phonon spectrum. For this reason they do not resonate with phonons, do not excite them and not lose energy over time $[2,3]$. DB can be characterized by two types of nonlinearity. In case of soft nonlinearity type frequency decreases with increasing amplitude, hard type of nonlinearity is characterized by growth of frequency with increasing DB amplitude.

Decreasing of DB mode frequency in case of soft nonlinearity type with growth of amplitude values can result in reaching the gap of phonon spectrum in case of its existence.

In a theoretical paper [38] it was shown that a onedimensional chain of atoms, interacting through one of the classical potentials (Born- Mayer, Lennard-Jones or Morse) is not able to support a DB with the hard type of nonlinearity. The authors were able to excite only the soft nonlinearity type $\mathrm{DB}$ considering a biatomic chain of atoms with a gap in the phonon spectrum. It was shown later in [39-46] that in two-dimensional and three-dimensional Morse crystals hard type of nonlinearity are able to exist as well. One can explain this possibility by the role of on-site potential presenting in crystals with higher dimensionality. The on-site potential suppresesses the effect of the increase of average interatomic distances in the DB localization center thus increasing the contribution of the hard core of interatomic potential by comparison to its soft tail.

The possibility of excitation of a moving DB in a closely packed raw of 2D Morse crystal was shown in [40].

One should mention that the configuration of the DB and its symmetry type depend on such factors as crystal lattice type and its dimensionality, interatomic bonds nature and mechanisms of DB initiation. However, not much works presenting some systematic description of possible types of $\mathrm{DB}$ able to exist in one crystal type can be found in literature. This work presents results of studying of DB of different symmetry type structure and localization degree which can be initiated in 2D Morse crystal by means of application of bell-shaped window to different phonon modes.

\section{Simulation details}

This work presents the results of comparison of two DB excited in 2D monoatomic Morse crystal. Both breathers have been initiated by means of excitation of phonon mode with a wave vector on the border of Brillouin zone in the non-linear regime. In case if the phonon mode frequency 

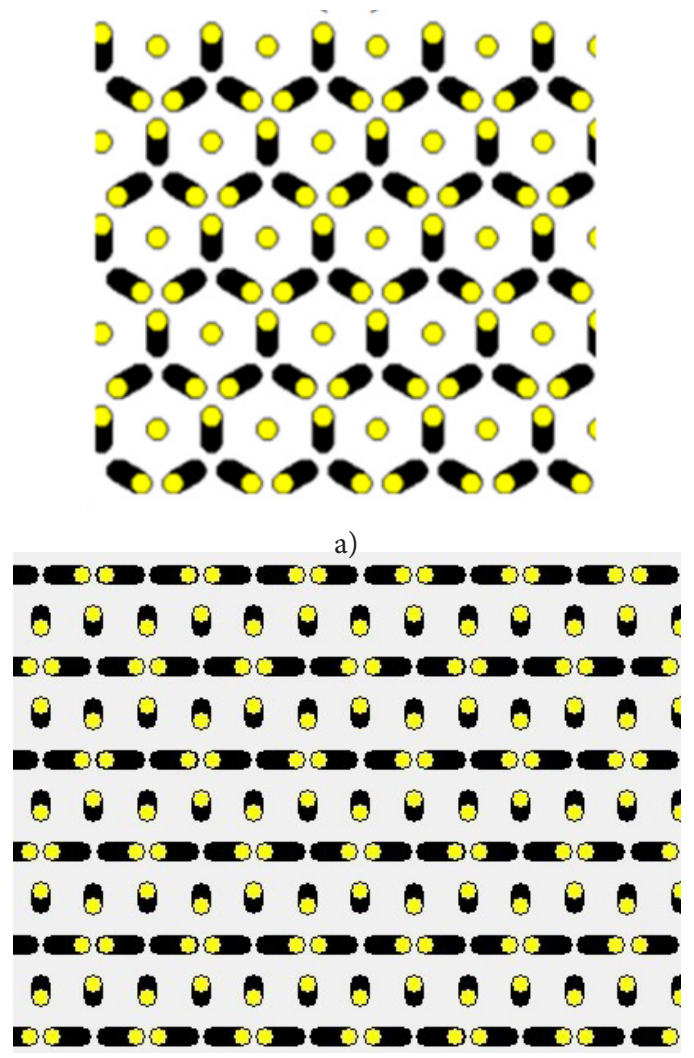

c)

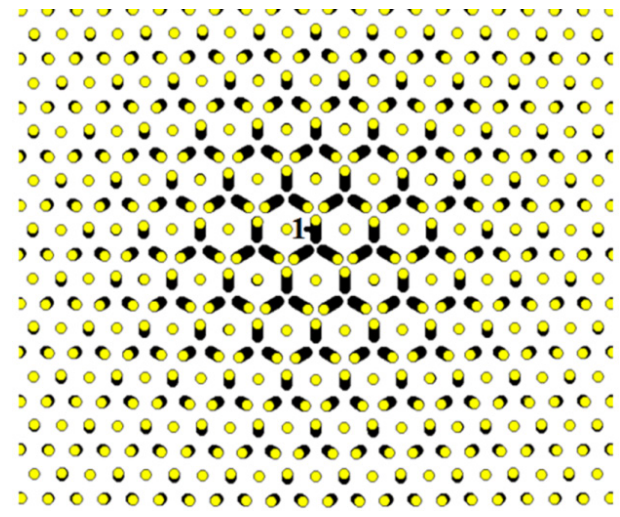

b)

3000000000000000000000 0000000000000000000000 3000000000000000000000 0000000000000000000000 30000000000000000000 0000000000000000000000 , 000000000000000000000 000000000000000000000 , 000000000000000000000 0000000000000000000000 3000000000000000000000 0000000000000000000000 3000000000000000000000 0000000000000000000000 3000000000000000000000

d)

Fig. 1. (Color online) (a) stroboscopic pattern of atomic motion of a the phonon mode of a high symmetry DB with a wave vector $\mathbf{q}=(\pi, \pi)$ on the border of Brillouin zone (b) stroboscopic pattern of atomic motion of a the phonon mode of a unsymmetrical DB (c) stroboscopic pattern of atomic movements in the vicinity of high symmetry DB that was initiated by means of superposition of bell-shaped function with the short wave phonon mode in the nonlinear regime. (d) stroboscopic pattern of atomic motions in the vicinity of unsymmetrical DB that was initiated by means of superposition of bell-shaped function with the short wave phonon mode in the nonlinear regime. Atomic shifts are increased by seven times.

grows with amplitude increase one can generate a spatially localized vibrational mode (namely the DB) by means of application of bell shaped function [49].

Both cases were analysed for the case of 2D crystal where those atoms are placed in the nodes of triangular lattice [49]. Interatomic interactions are defined by the long-range pairwise Morse potential.

$$
V(r)=D\left(e^{-2 \alpha\left(r-r_{m}\right)}-2 e^{-\alpha\left(r-r_{m}\right)}\right),
$$

where $r$ is the distance between couple of atoms, $D, \alpha, r_{m}-$ parameters defining the potential. The function $V(r)$ has the minimum at $r=r_{\mathrm{m}}$, corresponding to the minimum depth value (bond breaking energy) $D, \alpha$ is the parameter defining the bond stiffness.

Without loss of generality one can put $r_{\mathrm{m}}=1$ and $D=1$, selecting the appropriate unit of distance and energy. For the parameter $\alpha$ determining the rigidity of the interatomic bond the value $\alpha=5$ was considered. For the selected cut-off distance $5.5 a$, the equilibrium lattice parameter makes $a=0.98813$. Morse potential has been frequently used for simulation of different DB properties in crystals [23-29,40 - 46].

This crystal can support excited phonon modes with the wave vector on the Brillouin zone $\mathbf{q}=(\pi, \pi)$, shown on Figures 1 (a) and 1 (c). A computational cell containing $8 \times 8$ atoms and periodic boundary conditions was used for investigation of dynamics of mentioned modes.

Fig. 2 (a) shows the first mode frequency (Fig. 1 (a)) of the amplitude of atomic vibrations for cases when the size of the calculation cell was fixed (solid line) and when it was modified in order to maintain a zero external pressure (dashed line).

Correspondingly Fig. 2 (b) shows the dependence of the frequency of the second mode (Fig. 1 (c)) of the atomic oscillations amplitude for cases of fixed and variable computational cell size. The horizontal dashed line stands for the upper limit of the crystal phonon spectrum, $\omega_{\max }=2.995$. It can be seen that in case of constant volume both phonon modes in the nonlinear regime are characterized by frequency growth with amplitude followed by cleavage from the phonon spectrum upper limit. However in the zero external pressure case the mode frequency decreases with growing amplitude. One can thus conclude that DB can exist only in absence of breather induced local crystal "thermal expansion" and in case if their frequency will stay above the phonon spectrum.

Exponentially localized bell-shaped function having radial symmetry was applied for excision of high symmetry DB from flat phonon mode. The non-symmetrical DB has been obtained by means of superposition of two functions having different localization degree one solely in $x$ and second only in $y$ directions. The calculation was performed for a calculation cell containing $192 \times 192$ atoms for highly symmetrical DB and $80 \times 80$ for non-symmetrical DB with periodic boundary conditions for both cases.

The viscosity terms have been applied to the periphery of computational cells used for the absorption of smallamplitude waves emitted by the $\mathrm{DB}$ due to the initial conditions inaccuracy. Center of the bell function for highly 
symmetrical DB can be placed on one of the rest atoms or in a three neighboring vibrating atoms equidistant point. Both cases result in initiation of the third order symmetry DB. In the case of non-symmetrical DB the bell function center should only be arranged at a point between two atoms in a row. Because of the relatively low DB localization degree obtained in this study, it was revealed that the DB properties are very weakly dependent on its alignment.

\section{Results and discussion}

Fig. 2 shows the example of atomic displacements along $x$ axis for both types of breathers. It should be mentioned that the considered DB is not an exact solution of nonlinear equations of motion of atoms and should be regarded in the concept of quasibreathers [48], having a sufficiently long lifetime.

Dependence of DB frequency on the amplitude is presented on Fig. 3 by empty circles connected with a solid curve. Growth of DB frequency with increasing its amplitude is a witness of hard nonlinearity type.

However, the DB frequency growth rate with amplitudeincrease is much smaller than that of corresponding phonon mode. This fact can be explained by increase of average interatomic distances in the $\mathrm{DB}$ core that results in growth of contribution of the soft tail Morse potential to system dynamics by comparison to that of the phonon mode in

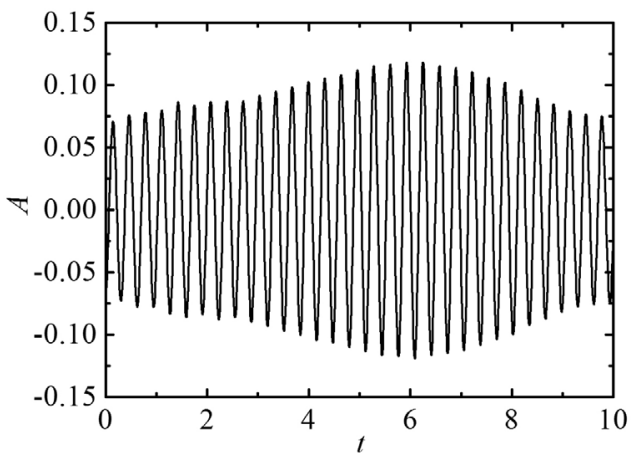

a) constant volume conditions.

Nonsymmetrical DB is defined by two bell-shaped function applied along $y$ axis and along the $x$ axis, which establishes its second order of symmetry, allowing DB to move along the closely packed atomic raw. In contrast to described case the high symmetry DB has lower localization degree and higher symmetry order that could be a probable reason of its immobility.

Unlike unbalanced DB [49], the breather considered has a high symmetry and a lower containment whereby, apparently cannot move through the crystal.

Investigations of both DB properties revealed their time stability within the calculations period $\left(10^{6}\right.$ oscillations) and retaining its initially given form with some negligible amplitude variations. Energy values of both $\mathrm{DB}$ possess the same order of magnitude.

\section{Conclusions}

We have studied two types of DB of different symmetry in two-dimensional monoatomic crystal with Morse interaction potential. Both DB were initiated by the excitation of shortwave phonon modes with following application of bellshape function. It was found that both types of $\mathrm{DB}$ are characterized by hard type of non-linearity and demonstrate the stability of properties over time. Amplitudes interval of $\mathrm{DB}$ existence in the case of high-symmetry $\mathrm{DB}$ about 2 times

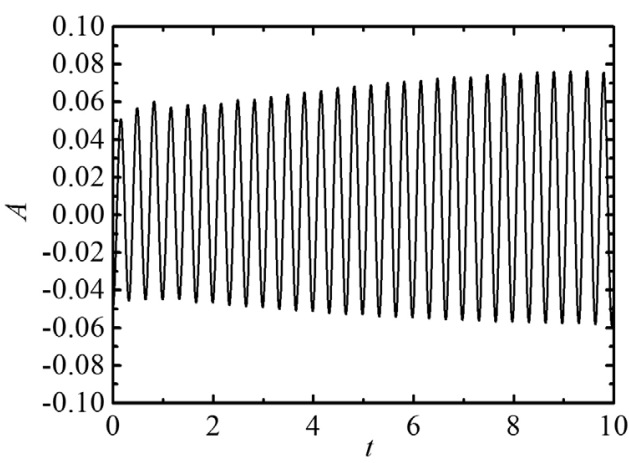

b)

Fig. 2. Displacement along $x$ direction for one central atom of the DB as the function of simulation time for the case of (a) High symmetry DB shown on Fig. 1 (a) and (b) nonsymmetrical DB presented on Fig. 1 (c).

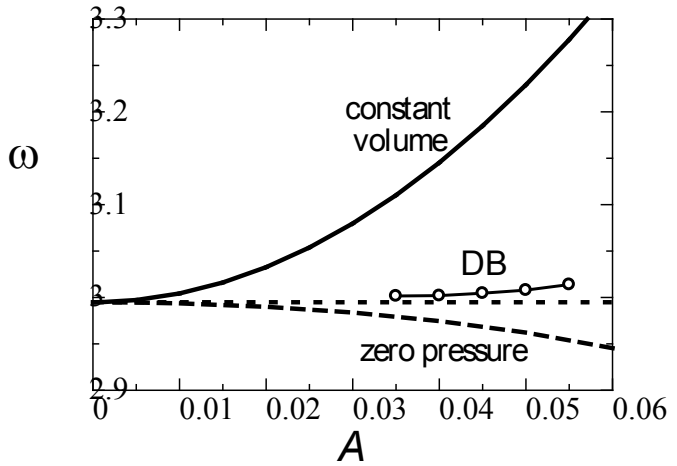

a)

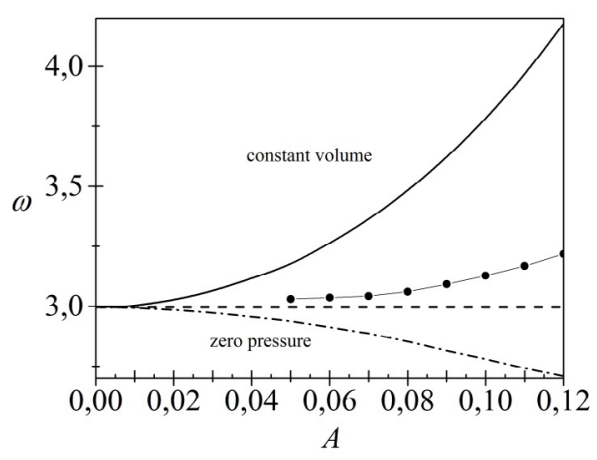

b)

Fig. 3. (a) The amplitude- frequency dependence of the phonon mode shown in Fig. 1 (a) in the nonlinear regime: solid line corresponds to calculations at constant volume, the dashed one goes for zero pressure case. (b) amplitude- frequency dependence of the phonon mode shown in Fig. 1 (c) of the amplitude in the nonlinear regime: solid line corresponds to calculations at constant volume, the dashed one goes for zero pressure case. The horizontal dashed line defines the upper limit of the phonon spectrum, $\omega_{\max }=2.995$. The empty circles connected by solid lines on both figures represent the calculated frequency of DB shown in Fig. 1 (a) and 1 (c) from the amplitude of its central atom vibrations. 
less than that for DB located in the closely-packed row. High symmetry and low degree of localization of the new type DB is the probable obstacle for its movement ability through the crystal.

Acknowledgements. This work was supported by the President of Russian Federation, the grant MK-5283.2015.2 for the young scientists support.

\section{References}

1. DolgovA.S.,Sov. Phys. SolidState.28, 907 (1986).

2. Sievers A. J., Takeno S., Phys. Rev. Lett. 61, 970 (1988).

3. Flach S., Gorbach A. V., Phys. Rep.467,1 (2008).

4. Voulgarakis N. K. et al.,Phys. Rev. B64, 020301 (2001).

5. Kalosakas G., Bishop A.R., Shreve A.P.,Phys. Rev. B66, 094303 (2002).

6. Campbell D. K., Flach S., Kivshar Y.S.,Phys. Today. 57, 43 (2004).

7. Manley M.E. et al.,Phys. Rev. B77, 214305 (2008).

8. Manley M.E. et al.,Phys. Rev. B 79, 134304 (2009)

9. Kempa M. et al.J. Phys.: Condens. Matter. 25, 055403 (2013).

10. Sievers A. J. et al.,Phys. Rev. B 88,104305 (2013).

11. Archilla J. F. R. et al.,Physica D 297, 56 (2015).

12. Chechin G. M., Lobzenko I. P., Letters on Materials 4, 226 (2014)

13. Chechin G. M., Dmitriev S. V., Lobzenko I. P., Ryabov D. S., Phys. Rev. B90, 045432 (2014).

14. Lobzenko I.P., Chechin G.M., Bezuglova G.S., Baimova Ju. A., Korznikova E. A., Dmitriev S. V., Phys. SolidState 201658 (3), 616 (2015) (in Russian).

15. Dmitriev S.V., Medvedev N.N., Mulyukov R.R., Pozhidaeva O.V., Potekaev A.I., Starostenkov M.D., Russian Physics Journal. 51, 858 (2008).

16. Khadeeva, L. Z., Dmitriev, S. V., Phys. Rev. B84, 144304 (2011).

17. Kiselev S. A., Sievers A. J., Phys. Rev. B 55, 5755 (1997).

18. Khadeeva L.Z., Dmitriev S.V., Phys. Rev. B81, 214306 (2010).

19. BaimovaJu.A., DmitrievS.V., KistanovA.A., PotekaevA.I., Izvestija vuzov fizika 56 (2), 60 (2013). (in Russian)

20. HaasM., HizhnyakovV., ShelkanA., KlopovM., SieversA.J., Phys. Rev. B84, 144303 (2011).

21. R.T. Murzaev, A.A. Kistanov, V.I. Dubinko, D.A. Terentyev, S. V. Dmitriev.,Comput. Mater. Sci. 98, 88 (2015).

22. Terentyev D.A., $\quad$ Dubinko A. V., Dubinko V.I., Dmitriev S.V., Zhurkin E.E., Sorokin M. V.,ModellingSimul. Mater. Sci. Eng. 23, 085007 (2015).

23. MedvedevN.N.,StarostenkovM.D., ManleyM.E., J. Appl. Phys. 114, 213506 (2013).

24. StarostenkovM.D., PotekaevA.I., DmitrievS.V., ZaharovP.V., EreminA.M., KulaginaV.V., Izvestija vuzov fizika 58 (9),136 (2015) (in Russian).

25. Medvedev N.N., Starostenkov M.D., Zaharov P.V., Pozhidaeva O.V., Technical Physics Letters 379 (3), 7 (2011) (in Russian).
26. MedvedevN.N., StarostenkovM.D., PotekaevA.I., ZakharovP.V., MarkidonovA.V., Eremin. A.M. Energy, RussianPhysicsJournal 57 (3), 387 (2014).

27. Zaharov P.V., Starostenkov M.D., Erjomin A.M., Markidonov A.V., Fundamental'nye problemy sovremennogo materialovedenija 11 (2), 260 (2014) (in Russian).

28. Zaharov P.V., Starostenkov M.D., Dmitriev S. V., Medvedev N. N., Eremin A. M., JETP Letters 148 (2), 252 (2015) (in Russian).

29. Zaharov P.V., Starostenkov M.D., Medvedev N.N., Erjomin A.M., Markidonov A.V., Fundamental'nye problemy sovremennogo materialovedenija 11 (3), 388 (2014) (in Russian).

30. Voulgarakis N.K., Hadjisavvas G., Kelires P.C., Tsironis G. P., Phys. Rev. B 69, 113201 (2004).

31. Yamayose Y., Kinoshita Y., Doi Y., Nakatani A., Kitamura T., Europhys. Lett. 80, 40008 (2007).

32. Doi Y., Nakatani A., J. Solid Mach. Mater. Eng. 6, 71 (2012).

33. HadeevaL.Z., DmitrievS.V., Kivshar'Ju.S., JETP Letters 97 (7), 580 (2011) (in Russian).

34. Korznikova E. A., Baimova J. A., Dmitriev S. V., Europhys. Lett. 102, 60004 (2013).

35. Baimova J.A., Dmitriev S. V., Zhou K., Europhys. Lett. 100, 36005 (2012).

36. Baimova J.A., Korznikova E.A., Lobzenko I.P., Dmitriev S. V., Rev. Adv. Mater. Sci. 42, 68 (2015).

37. Liu B., Baimova J.A., Dmitriev S. V., Wang X., Zhu H., Zhou K., J. Phys. D: Applied Physics. 46, 305302 (2013).

38. Kiselev S. A., Bickham S. R., Sievers A. J., Phys. Rev. B 48, 13508 (1993).

39. Dmitriev S.V., Kistanov A.A., Dubinko V.I., Springer Series in Materials Science. 221, 205 (2015).

40. Kistanov A.A., Murzaev R.T., Dmitriev S.V., Dubinko V.I., Hizhnjakov V.V.,., JETP Letters 99, 403 (2014) (in Russian).

41. KistanovA.A.,KorznikovaE.A., FominS.Yu., ZhouK., Dmitriev S. V., Letters on materials 4 (4), 315 (2014).

42. Kistanov A.A., Dmitriev S. V., Chetverikov A.P., Velarde M.G., The European Physical Journal B 87 (9), 211 (2014).

43. Semenov A.S., Korznikova E.A., Dmitriev S. V., Letters on materials. 5 (1), 11 (2015) (in Russian).

44. Kistanov A.A., Zhou K., Korznikova E. A., Fomin S. Ju., Dmitriev S. V., Fundamental'nye problemy sovremennogo materialovedenija 12 (1),103 (2015). (in Russian)

45. Kistanov A.A., Semenov A.S., Dmitriev S.V., JETP Letters 146 (4), 869 (2014) (in Russian).

46. Kistanov A.A., Dmitriev S.V., Semenov A.S., Dubinko V.I., Terent'ev D. A., Technical Physics Letters 40 (15), 58 (2014) (in Russian).

47. Timoshenko S.P., Gud’er Dzh. Teorija uprugosti, 576 (1975) (in Russian).

48. Chechin G. M., Dzhelauhova G.S., Mehonoshina E.A., Phys. Rev., E 74, 036608 (2006).

49. Korznikova E.A., Fomin S. Ju., Soboleva Je.G., Dmitriev S. V.. JETP Letters, 103 (4), 303 - 308 (2016) (in Russian). 